\section{Refractory ulcerative colitis complicated by cytomegalovirus infection successfully treated with valganciclovir}

\author{
Tiziana Larussa, ${ }^{1}$ Evelina Suraci, ${ }^{1}$ \\ Immacolata Nazionale, ${ }^{1}$ \\ Francesco Conforti, ${ }^{2}$ Maria Imeneo, ${ }^{1}$ \\ Francesco Luzza ${ }^{1}$ \\ Departments of ${ }^{1} \mathrm{Health}$ Science and \\ 2Pathological Anatomy, University \\ of Catanzaro Magna Graecia, Catanzaro, \\ Italy
}

\begin{abstract}
Cytomegalovirus (CMV) infection is widespread in the general population. In patients with severe and/or steroid-refractory ulcerative colitis (UC), local reactivation of CMV can be detected in actively inflamed colonic tissue in approximately $30 \%$ of cases. However, the role of CMV in patients with UC is not clearly understood. There is evidence to show a possible role in exacerbating a colitis flare, whereas other studies describe CMV as an innocent bystander. We report the case of a patient with severe UC complicated by CMV infection who did not respond to conventional therapy. A complete diagnostic panel for CMV diagnosis, including tissue polymerase chain reaction and immunohistochemistry, was carried out. Three-week therapy with oral valganciclovir resulted in dramatic clinical and endoscopic improvement. Timing of diagnosis and treatment of CMV infection complicating UC is crucial in order to recognize the organ-disease and plan appropriate treatment.
\end{abstract}

\section{Introduction}

Cytomegalovirus (CMV) infection is widespread in the general population with a prevalence of $40-100 \%{ }^{1}$ In the immunocompetent host, primary infection can result in EpsteinBarr virus-like mononucleosis syndrome. T cells control viral replication and disease but do not obtain complete eradication, leading to viral latency. In immunologically impaired patients, the virus may cause serious multiorgan involvement and complications due to the CMV-associated disease both in cases of primary infection and, the most frequent event, reactivation. $^{2}$

In patients with severe and/or steroidrefractory ulcerative colitis (UC), local reacti- vation of CMV can be detected in actively inflamed colonic tissue in approximately $30 \%$ of cases. ${ }^{3}$ The role of CMV in patients with inflammatory bowel disease (IBD) is not clearly understood. There is evidence to show a role in exacerbating a colitis flare, whereas other studies describe CMV as an innocent bystander. ${ }^{4}$ Furthermore, patients with IBD are frequently treated with immunosuppressive agents which may increase infection/reactivation risk while inflammation itself is a predisposing factor for infection.

In this study, we report a case of refractory UC complicated by CMV colitis successfully treated with oral valganciclovir.

\section{Case Report}

A 64-year old male with a diagnosis of left UC in the previous eight months came to our observation reporting a 2-month history of repeated hospital admissions for colitis relapse. Disease status until then had been good and the patient had maintained remission with oral and topical mesalamine. Two months before UC diagnosis, he had a heart attack and underwent multiple coronary stenting requiring antiplatelet therapy (clopidrogrel $75 \mathrm{mg} /$ day).

During the previous periods of hospitalization, he had received intravenous steroid therapy (methylprednisolone $1 \mathrm{mg} / \mathrm{kg} /$ day) continuing with this treatment for more than 40 days (although dose scheduling was not optimal) without clinical improvement. After completing this period of steroid therapy without receiving any benefit, azathioprine was also introduced at $0.85 \mathrm{mg} / \mathrm{kg} / \mathrm{day}$ but this was suspended at day 4 of treatment due to pancytopenia. Furthermore, iatrogenic diabetes has developed.

The patient was admitted to our hospital a few days after an adverse reaction to azathioprine and presented with normal vital signs. He reported bloody diarrhea occurring 4-5 times a day, abdominal pain and weakness. On physical examination, his abdomen was slightly distended with normoactive bowel sounds but no tenderness on deep palpation. Laboratory tests were remarkable for a slight decrease in the white blood cell count $\left(4.12 \times 10^{3} / \mathrm{uL}\right.$; normal range $\left.5.2-12.4 \times 10^{3} / \mathrm{uL}\right)$ without formula alteration, in spite of the prolonged use of steroids that are likely to determine increased white blood cell levels. This slight leukopenia agreed with the recent bone marrow toxicity developed after thiopurine therapy. Red blood cells were decreased (3.60×106/uL; normal range 4.7-6.1 $\left.\times 10^{6} / \mathrm{uL}\right)$ as were hemoglobin $(10.4 \mathrm{~g} / \mathrm{dL}$; normal range 14 $18 \mathrm{~g} / \mathrm{dL})$, potassium serum level $(2.8 \mathrm{mmol} / \mathrm{L}$; normal range $3.5-5.1 \mathrm{mmol} / \mathrm{L})$, albumin $(2.8$
Correspondence: Francesco Luzza, Department of Health Science, University of Catanzaro Magna Graecia, Campus di Germaneto, viale Europa, 88100 Catanzaro, Italy.

Tel. +39.0961.3647111 - Fax: +39.0961 .3647164$

E-mail: luzza@unicz.it

Key words: ulcerative colitis, cytomegalovirus, valganciclovir, ganciclovir.

Acknowledgments: the authors would like to thank Dr. Benedetto Caroleo (Infectious Disease Unit, University of Catanzaro Magna Graecia, Italy) for his helpful advice.

Contributions: TL wrote the manuscript; ES and IN collected patient's clinical information; MI and FC analyzed and discussed clinical and histopathological data; FL revised the paper.

Conflict of interests: the authors report no conflict of interests.

Received for publication: 3 August 2011.

Revision received: 12 June 2012.

Accepted for publication: 10 July 2012.

This work is licensed under a Creative Commons Attribution NonCommercial 3.0 License (CC BYNC 3.0).

(C) Copyright T. Larussa et al., 2012

Licensee PAGEPress, Italy

Gastroenterology Insights 2012; 4:e19

doi:10.4081/gi.2012.e19

$\mathrm{g} / \mathrm{dL}$; normal range $3.4-4.8 \mathrm{~g} / \mathrm{dL}$ ), and iron (28 $\mu \mathrm{g} / \mathrm{dL}$; normal range $61-157 \mu \mathrm{g} / \mathrm{dL}$ ). C-reactive protein and erythrocyte sedimentation rate were increased $(30 \mathrm{mg} / \mathrm{L}$; normal range $0-5$ $\mathrm{mg} / \mathrm{L}$, and $35 \mathrm{~mm} / \mathrm{h}$; normal value up to 30 $\mathrm{mm} / \mathrm{h}$, respectively). Renal, pancreatic and liver function tests were unremarkable. Abdominal X-ray revealed a normal bowel gas pattern and no evidence of colonic dilatation or air-fluid levels. At ultrasound evaluation, no alterations in liver, spleen, gallbladder or pancreas were found. We started intravenous therapy with methylprednisolone at $1 \mathrm{mg} / \mathrm{kg} / \mathrm{day}$ and parenteral supplementation with albumin, iron, potassium and folic acid. Since hemoglobin levels decreased up to $8 \mathrm{~g} / \mathrm{dL}$ a blood transfusion was provided. It should be noted that the patient required insulin for the persistence of iatrogenic diabetes. No significant clinical improvement was observed over the following five days of hospitalization. Thereafter, a colonoscopy with ileoscopy was performed which displayed multiple deep ovalar and serpiginous ulcers with undeterrmined margins and a number of pseudopolyps throughout the colon. No lesions were observed in the terminal ileum explored for $20 \mathrm{~cm}$.

In this context of refractoriness to conventional high-dose therapy and prolonged 
immune-suppressive patient status, before considering alternative medical strategies (i.e. cyclosporine, infliximab, colectomy), the endoscopic pattern moved our clinical suspicions to another cause of persistent severe colitis, such as CMV infection.

Specific serum IgG but not IgM to CMV were detected. Nevertheless, leukocyte polymerase chain reaction (PCR) showed CMV viremia. Blood sample copy number of CMV DNA was 284 copies/mL. Furthermore, biopsy specimens obtained from the margin and base of the ulcers showed typical CMV nuclear inclusion bodies (Hematoxylin and Eosin staining) and DNA amplification by PCR on colonic tissue was positive for CMV replication, with a copy number of CMV DNA of 10 copies/mg.

These findings indicated that $\mathrm{CMV}$ reactivation was associated with UC exacerbation. Methylprednisolone was, therefore, gradually tapered, and valganciclovir (450 mg twice daily) was administered orally for three weeks. Following the initiation of antiviral therapy, abdominal symptoms gradually resolved and laboratory tests slowly returned to normal range. After three weeks of valganciclovir, a sigmoidoscopy was performed which showed a dramatic improvement in the endoscopic lesions. At the same time, serum CMV-DNA was no longer detected and PCR on colonic tissue resulted negative for CMV replication. Hematoxylin and eosin staining performed on biopsy samples showed no typical inclusions suggestive of CMV organ-disease. The patient stopped antiviral therapy and was discharged with oral mesalamine $3.2 \mathrm{gr} / \mathrm{day}$.

\section{Discussion and Conclusions}

In the present case, we describe a superinfection with cytomegalovirus occurring in an immune-compromised host with UC. Treatment failure after five days of intravenous steroid therapy and endoscopic find-

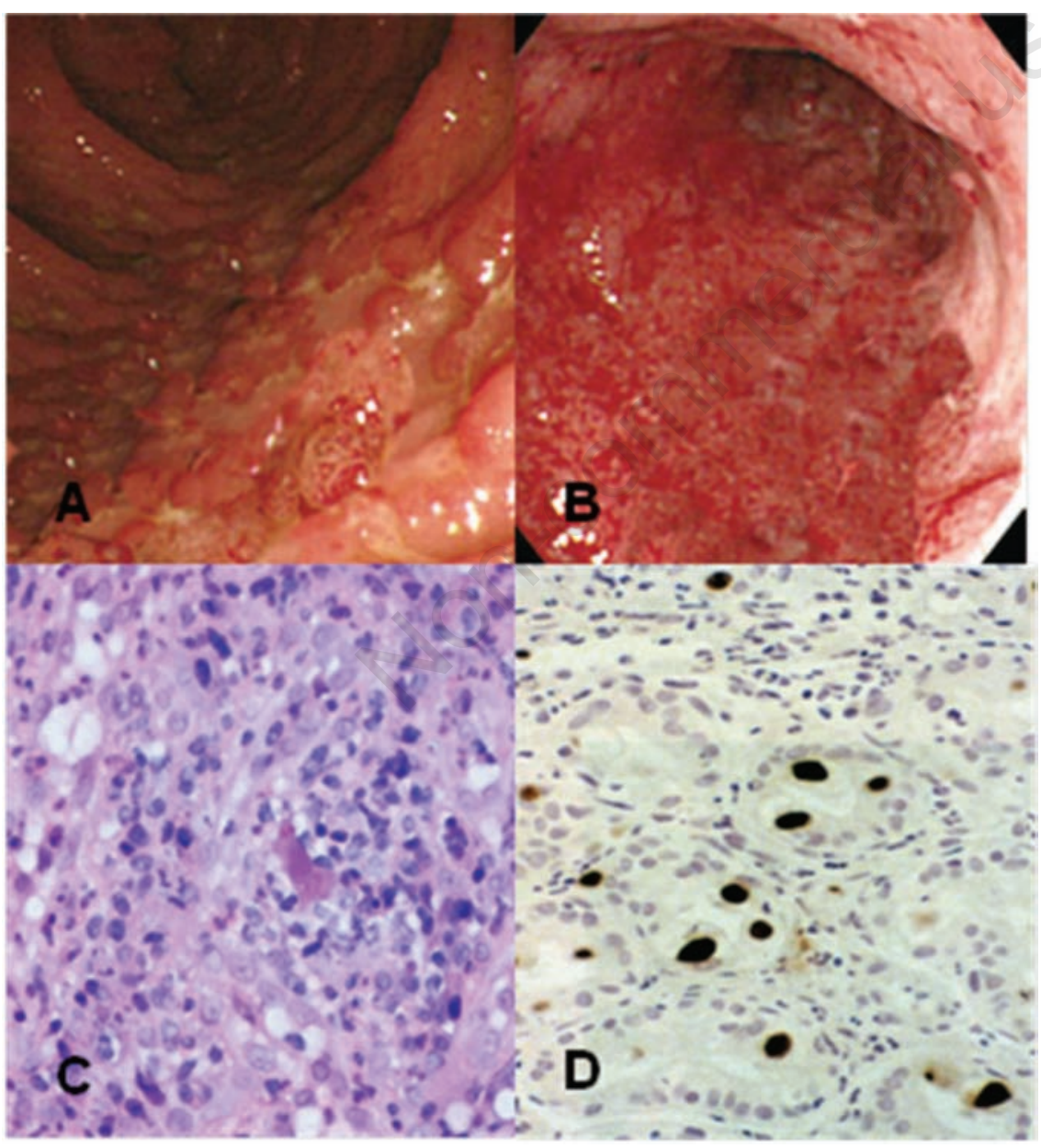

Figure 1. Endoscopic (A and B) and histological (C) hematoxylin and eosin and (D) immunohistochemistry findings on colonic endoscopic biopsies taken from a patient with refractory ulcerative colitis complicated by cytomegalovirus infection, before (A, C and $D$ ) and after (B) antiviral treatment with oral valganciclovir. Immunostains of cytomegalovirus infection show a large number of immunoreactive epithelial cells. ings suggested CMV infection in addition to UC. This was confirmed by leukocyte and colonic tissue PCR positivity along with the presence of cytomegalic cells at Hematoxylin and Eosin staining (Figure 1).

Patients with IBD are at higher risk of CMV infections due to the frequent use of immunosuppressive therapies, the particular tropism of CMV for sites of inflammation and the altered immunological state which is consistent with the molecular pathogenesis of intestinal disease. ${ }^{5}$ Given the association of CMV colitis with refractory UC, CMV infection should be ruled out with rectal biopsy and serology whenever a patient presenting with UC exacerbation does not respond to conventional therapies. In particular, we must consider a possible CMV colitis when patients in remission or with mild-stable symptoms unexpectedly develop a severe or atypical exacerbation, complicated by steroid-refractoriness. ${ }^{6}$

As shown in Table 1, there are several methods for detecting CMV disease. Histological examination is a relatively easy approach, but sensitivity is not high (10-87\%). In this study, antigenemia pp65 was not performed given that it has been demonstrated that it is not predictive of underlying CMV detection in colonic tissue of patients with IBD. In fact, it is largely being replaced by the introduction of leukocyte PCR which provides better quantification of viral presence. ${ }^{7} \mathrm{~A}$ combination of the above listed methods may increase the CMV detection rate. ${ }^{8}$

The most recent European Crohn's and Colitis Organization guidelines (ECCO) recommend tissue PCR or immunoistochemestry to investigate CMV in immunomodulatorrefractory cases of IBD. ${ }^{9}$ Furthermore, the role of endoscopy in patients with UC complicated by CMV infection has been recently addressed. ${ }^{10}$ So, specific endoscopic findings, such as those reported in this study, may facilitate the early recognition of CMV colitis. As regards antiviral therapy, CMV colitis is usually treated

Table 1. Tests used to detect cytomegalovirus infection and results in a patient with ulcerative colitis.

\begin{tabular}{lc}
\hline Methods & Results \\
Serum IgG & + \\
Serum IgM & - \\
\hline Leukocyte PCR & + \\
H\&E & + \\
\hline IHC & + \\
Colonic tissue PCR & + \\
\hline Antigenemia pp65 & Not performed \\
\hline Ig, Immunoglobulin; H\&E, Hematoxylin and Eosin; IHC, immunois- \\
tochemestry (both on colonic tissue); PCR, polymerase chain reaction.
\end{tabular}


with ganciclovir, although foscarnet, valganciclovir, and cidofovir have also been suggested. Any benefits of antiviral therapy must outweigh the risks associated with the medication used. ${ }^{11}$ According to ECCO guidelines, treatment with antivirals is recommended when CMV is detected in colonic tissue. However, intravenous administration of ganciclovir requires hospitalization; therefore, we preferred to use valganciclovir for outpatient management of the disease.

CMV infection in UC patients, especially in those who are immune-compromised by steroid therapy, can produce severe systemic disease and often leads to colectomy. Particularly severe and unrecognized cases may display an even poorer clinical outcome. Clinicians should evaluate what are the best methods and timing needed to control the infection, in order to recognize the so-called organ-disease and plan appropriate treatment. It is becoming clear that early diagnosis would help to prevent the worst consequences. Clinicians should be aware of the importance of considering CMV infection in a setting of UC when clinical and laboratory data are suggestive of refractory colitis.

\section{References}

1. Zhang LJ, Hanff P, Rutherford C, et al. Detection of human cytomegalovirus DNA, RNA, and antibody in normal donor blood. J Infect Dis 1995;171:1002-6.

2. Crough T, Khanna R. Immunobiology of human cytomegalovirus: from bench to bedside. Clin Microbiol Rev 2009;22:76-98.

3. Ayre K, Warren BF, Jeffery K, Travis S. The role of CMV in steroid-resistant ulcerative colitis: a systematic review. J Crohn's Colitis 2009;3:141-8.

4. Lawlor G, Moss AC. Cytomegalovirus in inflammatory bowel disease: pathogen or innocent bystander? Inflamm Bowel Dis 2010;16:1620-7.

5. Papadakis KA, Tung JK, Binder SW, et al. Outcome of cytomegalovirus infections in patients with inflammatory bowel disease. Am J Gastroenterol 2001;96:2137-42.

6. Rahier JF, Yazdanpanah Y, Colombel JF, Travis SPL. The European (ECCO) consensus on infection in IBD: what does it change for the clinician? Gut 2009;58: 1313-5.
7. Landry ML, Ferguson D. Comparison of quantitative citomegalovirus antigenemia assay with culture methods and correlation with clinical disease. J Clin Microbiol 1993;31:2851-6.

8. de la Hoz RE, Stephens G, Sherlock C. Diagnosis and treatment approaches of CMV infections in adult patients. J Clin Virol 2002;25Suppl2:S1-12.

9. Rahier JF, Ben-Horin S, Chowers Y, et al. on behalf of the European Crohn's and Colitis Organisation (ECCO). European evidence-based consensus on the prevention, diagnosis and management of opportunistic infections in inflammatory bowel disease. J Crohn's Colitis 2009; 3:47-91.

10. Suzuki H, Kato J, Kuriyama M, et al. Specific endoscopic features of ulcerative colitis complicated by cytomegalovirus infection. World J Gastroenterol 2010;16: 1245-51.

11. Markham A, Faulds D. Ganciclovir. An update of its therapeutic use in cytomegalovirus infection. Drugs 1994;48: 455-84. 